\title{
MARTINGALE REPRESENTATION IN UNIFORMLY CONVEX SPACES
}

\author{
D. LANDERS AND L. ROGGE
}

\begin{abstract}
In this paper we define the concept of a martingale in a uniformly convex Banach space and show that each bounded martingale is convergent and can be represented as a sequence of nearest point projections onto closed convex sets of one element of the Banach space. This result contains classical representation theorems for usual martingales and yields applications in the theory of nearest point projections in $L_{p}$ spaces.
\end{abstract}

1. Notations. Let $B$ be a Banach space with norm \|\| . If $x \in B, C \subset B$, let $d(x, C)=\inf \{\|x-y\|: y \in C\}$

The Banach space $B$ is uniformly convex iff for each $\varepsilon>0$ there exists $\delta(\varepsilon)>0$ such that $\left\|x_{1}\right\| \leqslant 1,\left\|x_{2}\right\| \leqslant 1,\left\|\frac{1}{2}\left(x_{1}+x_{2}\right)\right\| \geqslant 1-\delta(\varepsilon)$ implies $\left\|x_{1}-x_{2}\right\| \leqslant \varepsilon$.

For the sake of completeness we cite the following result, which can easily be derived from the considerations in [1, pp. 113-114].

REMARK. Let $B$ be a uniformly convex Banach space and $r>0$ be fixed. Then for each $\varepsilon>0$ there exists $\delta(\varepsilon)>0$ such that for all convex sets $C \subset B$ with $0 \in C$ we have: $\|x\| \leqslant r, y_{1}, y_{2} \in C$ and $\left\|x-y_{1}\right\|,\left\|x-y_{2}\right\| \leqslant d(x, C)$ $+\delta(\varepsilon)$ imply $\left\|y_{1}-y_{2}\right\| \leqslant \varepsilon$.

In a uniformly convex space $B$ for each $x \in B$ and each closed convex set $C \subset B$ there exists a unique projection $\pi(x \mid C) \in C$ of $x$ onto $C$, i.e. a unique element $\pi(x \mid C) \in C$ such that $\|x-\pi(x \mid C)\|=d(x, C)$.

Definition. We call $x_{n} \in B, n \in \mathbf{N}$, a martingale with respect to the closed convex sets $C_{n} \subset B, n \in \mathbf{N}$, iff

(i) $0 \in C_{m} \subset C_{n}$ for $m \leqslant n$,

(ii) $x_{m}=\pi\left(x_{n} \mid C_{m}\right)$ for $m \leqslant n$.

If $B$ is a Hilbert space this is the definition of Brunk [3].

If $B$ is an $L_{p}(\Omega, Q, P)$, where $(\Omega, Q, P)$ is a probability space, then important examples for projections onto closed convex sets are the classical conditional expectations, the conditional expectations given a $\sigma$-lattice (see [2], [3]), the $p$-predictors in the sense of Ando-Amemiya (see [1]) or the conditional $p$-means in the sense of Brunk (see [4]).

2. The result. Now we give a representation theorem for martingales, which generalizes Brunk's representation theorem (see [3]) from Hilbert spaces to

Received by the editors December 27, 1977 and, in revised form, July 17, 1978.

AMS (MOS) subject classifications (1970). Primary 60G45; Secondary 41 A50.

Key words and phrases. Martingale, uniformly convex Banach space, conditional expectation. 
uniformly convex Banach spaces. We remark that Brunk's methods are closely adjusted to a Hilbert space and cannot be applied for uniformly convex Banach spaces.

TheOREM. Let $B$ be a uniformly convex Banach space. Let $x_{n} \in B, n \in \mathbf{N}$, be a martingale with respect to the closed convex sets $C_{n} \subset B, n \in \mathbf{N}$. Assume that $\sup \left\|x_{n}\right\|<\infty$. Then $x_{n}, n \in \mathbf{N}$, converges strongly to an element $x \in B$ and $x_{n}=\pi\left(x \mid C_{n}\right), n \in \mathbf{N}$.

Proof. Since a uniformly convex Banach space is reflexive and in a reflexive Banach space each bounded set is relatively weakly sequentially compact there exists a subsequence $x_{n}, n \in \mathbf{N}_{0}$, converging weakly to an element $x \in B$.

Since $\sup _{n \in \mathrm{N}}\left\|x_{n}\right\|<\infty$, we may w.l.g. assume that $\lim _{n \in N_{0}}\left\|x_{n}-x\right\|=a$, $a \in \mathbf{R}$.

We prove at first that

$$
x_{n}, n \in \mathrm{N}_{0} \text {, is Cauchy convergent. }
$$

As for convex sets weak closure and strong closure coincide and $x$ is in the weak closure of the convex set $\cup_{n \in \mathrm{N}} C_{n}$, there exists a sequence $z_{n} \in C_{n}$ with $\left\|z_{n}-x\right\|_{n \in N} \underset{n}{\rightarrow}$. Since $x_{n}=\pi\left(x_{m} \mid C_{n}\right)$ for $m>n$ and $z_{n} \in C_{n}$ we obtain

$$
\left\|x_{m}-x_{n}\right\| \leqslant\left\|x_{m}-z_{n}\right\| \quad \text { if } m \geqslant n .
$$

Let $\varepsilon>0$ be fixed and $\delta=\delta(\varepsilon)$ be chosen according to our Remark with $r:=\sup _{n \in \mathrm{N}}\left\|x_{n}\right\|$. As $\left\|z_{n}-x\right\| \underset{n \in \mathbf{N}}{\rightarrow} 0$, (1) implies that there exists $n_{0}(\varepsilon) \in \mathbf{N}$ such that

$$
\left\|x_{m}-x_{n}\right\| \leqslant\left\|x_{m}-x\right\|+\delta / 4 \quad \text { if } m>n \geqslant n_{0}(\varepsilon) .
$$

Since $\left\|x_{m}-x\right\| \underset{m \in N_{0}}{\rightarrow} a$, according to (2) there exists $n_{1}(\varepsilon) \geqslant n_{0}(\varepsilon)$ such that

$$
\left\|x_{m}-x_{n}\right\| \leqslant a+\delta / 2 \quad \text { if } m>n \geqslant n_{1}(\varepsilon), m \in \mathbf{N}_{0} \text {. }
$$

Since $x_{m}, m \in \mathbf{N}_{0}$, converges weakly to $x$, we have for each $n \in \mathbf{N}$ that $x_{m}-x_{n}, m \in \mathbf{N}_{0}$, converges weakly to $x-x_{n}$ and hence

$$
\left\|x-x_{n}\right\| \leqslant \frac{\lim }{m \in \mathbf{N}_{0}}\left\|x_{m}-x_{n}\right\| .
$$

Therefore for each $n \in \mathbf{N}$ there exists $k_{n}(\varepsilon) \in \mathbf{N}$ such that

$$
\left\|x-x_{n}\right\|-\delta / 4 \leqslant\left\|x_{m}-x_{n}\right\| \quad \text { if } m>k_{n}(\varepsilon), m \in \mathbf{N}_{0} \text {. }
$$

Since $\left\|x-x_{n}\right\| \underset{n \in N_{0}}{\rightarrow} a$, we obtain from (3) and (5) that there exists $n_{2}(\varepsilon) \geqslant$ $n_{1}(\varepsilon)$ such that

$$
\begin{aligned}
a-\delta / 2 \leqslant\left\|x_{m}-x_{n}\right\| & \leqslant a+\delta / 2 \\
\text { if } m>n & \geqslant n_{2}(\varepsilon), m>k_{n}(\varepsilon) \text { and } m, n \in \mathbf{N}_{0} .
\end{aligned}
$$

Now let $s_{1}, s_{2} \in \mathbf{N}_{0}$ with $s_{2}>s_{1}>n_{2}(\varepsilon)$ and choose $m \in \mathbf{N}_{0}$ with $m>$ $\max \left(n_{2}(\varepsilon), k_{s_{1}}(\varepsilon), k_{s_{2}}(\varepsilon)\right)$. Then (6) implies that

$$
\left\|x_{m}-x_{s_{1}}\right\| \leqslant\left\|x_{m}-x_{s_{2}}\right\|+\delta=d\left(x_{m}, C_{s_{2}}\right)+\delta \text {. }
$$


Since $\left\|x_{m}-x_{s_{2}}\right\|=d\left(x_{m}, C_{s_{2}}\right)$ and $x_{s_{1}}, x_{s_{2}} \in C_{s_{2}}$ we obtain from (7) according to our Remark that

$$
\left\|x_{s_{1}}-x_{s_{2}}\right\|<\varepsilon \quad \text { if } s_{1}, s_{2} \in \mathbf{N}_{0}, s_{1}, s_{2}>n_{2}(\varepsilon) \text {. }
$$

This implies (*). Hence $\left\|x_{n}-x\right\| \underset{n \in \mathbf{N}_{0}}{\rightarrow} 0$, because $x_{n}, n \in \mathbf{N}_{0}$, converges weakly to $x$. Therefore $x_{n}=\pi\left(x \mid C_{n}\right)$ for all $n \in \mathrm{N}$, as $x_{n}=\pi\left(x_{m} \mid C_{n}\right), n<m$, and the projection operator is continuous. Since $x$ is in the strong closure of $\cup_{n \in \mathrm{N}} C_{n}$, we immediately obtain that $x_{n}, n \in \mathrm{N}$, converges strongly to $x$.

We remark that obviously the preceding result also holds true for martingales, indexed by a net.

Our theorem yields classical martingale representation theorems or Brunk's representation theorem and is moreover applicable to martingales of $p$ predictors or martingales of $p$-means.

\section{REFERENCES}

1. T. Ando and I. Amemiya, Almost everywhere convergence of prediction sequence in $L_{p}$ $(1<p<\infty)$, Z. Wahrscheinlichkeitstheorie und Verw. Gebiete 4 (1965), 113-120.

2. R. E. Barlow, D. J. Bartholomew, J. M. Bremner and H. D. Brunk, Statistical inference under order restrictions, Wiley, London, New York, 1970.

3. H. D. Brunk, Conditional expectations given a o-lattice and applications, Ann. Math. Statist. 36 (1965), 1339-1350.

4. Uniform inequalities for conditional p-means given o-lattices, Ann. Probability 3 (1975), 1025-1030.

5. J. Neveu, Discrete parameter martingales, North-Holland, Amsterdam, 1975.

6. M. M. Rao, Inference in stochastic processes. III, Z. Wahrscheinlichkeitstheorie und Verw. Gebiete 8 (1967), 49-72.

MATHEMATISChes INSTITUT der UNIVERSitÄt ZU KölN, Weyertal 86-90, D-5000 KölN 41, Federal Republic of Germany

Universtït Konstanz, Fachibereich WiRtschafts-Wissenschaften/Statistik, Postfach 7733, D-7750 Konstanz, Federal Republic of Germany 